\title{
QoS management and control for an all-IP WiMAX network architecture: Design, implementation and evaluation
}

\author{
Thomas Michael Bohnert ${ }^{\mathrm{a}, *}$, Marco Castrucci ${ }^{\mathrm{b}}$, Nicola Ciulli ${ }^{\mathrm{c}}$, Giada Landi ${ }^{\mathrm{c}}$, \\ Ilaria Marchetti ${ }^{\mathrm{b}}$, Cristina Nardini ${ }^{\mathrm{b}}$, Bruno Sousa ${ }^{\mathrm{e}}$, Pedro Neves ${ }^{\mathrm{d}}$ and Paulo Simoes ${ }^{\mathrm{e}}$ \\ ${ }^{a}$ Siemens Corporate Technology, Information and Communication IC 2, Munich, Germany \\ ${ }^{\mathrm{b}}$ University of Rome "Sapienza", Roma, Italy \\ ${ }^{\mathrm{c}}$ Nextworks, Pisa, Italy \\ ${ }^{\mathrm{d}}$ Portugal Telecom Inovação, Aveiro, Portugal \\ ${ }^{\mathrm{e}}$ University of Coimbra, DEI, Pinhal de Marrocos, Polo II, Coimbra, Portugal
}

\begin{abstract}
The IEEE 802.16 standard provides a specification for a fixed and mobile broadband wireless access system, offering high data rate transmission of multimedia services with different Quality-of-Service (QoS) requirements through the air interface. The WiMAX Forum, going beyond the air interface, defined an end-to-end WiMAX network architecture, based on an all-IP platform in order to complete the standards required for a commercial rollout of WiMAX as broadband wireless access solution. As the WiMAX network architecture is only a functional specification, this paper focuses on an innovative solution for an end-to-end WiMAX network architecture offering in compliance with the WiMAX Forum specification. To our best knowledge, this is the first WiMAX architecture built by a research consortium globally and was performed within the framework of the European IST project WEIRD (WiMAX Extension to Isolated Research Data networks). One of the principal features of our architecture is support for end-to-end QoS achieved by the integration of resource control in the WiMAX wireless link and the resource management in the wired domains in the network core. In this paper we present the architectural design of these QoS features in the overall WiMAX all-IP framework and their functional as well as performance evaluation. The presented results can safely be considered as unique and timely for any WiMAX system integrator.
\end{abstract}

Keywords: WiMAX, QoS, resource management and control, WiMAX network reference model, NSIS signalling, session imitation protocol, WEIRD

\section{Introduction}

IEEE 802.16 standard defines one of the most promising technologies for Broadband Wireless Access, providing high performance in terms of data rate and coverage. In particular, it defines an air interface specification for fixed (version "d" [9]) and mobile (version "e" [8]) Metropolitan Area Networks (MANs), specifying a common Medium Access Control (MAC) protocol with different physical layer specifications dependent on the used spectrum. The 802.16 MAC protocol is designed to support a wide

\footnotetext{
${ }^{*}$ Corresponding author: Thomas Michael Bohnert, Siemens Corporate Technology, Information and Communication IC 2, Otto-Hahn-Ring 6, 81739 Munich, Germany. E-mail: tmb@nginet.de.
} 
range of communication services (data, voice, and video), providing support for continuous and bursty traffic, Quality of Service (QoS) mechanisms and methods of bandwidth allocation.

One distinct feature of IEEE 802.16 is the explicit integration of QoS mechanisms over a connectionoriented transaction design. Each packet transmitted over the air interface between the Subscriber Station (SS) and the Base Station (BS) is associated with an uplink or downlink Service Flow (SF) that specifies the scheduling class and the related QoS parameters, like maximum and minimum rate, jitter and priority. The IEEE 802.16e specification defines five different scheduling services:

- Unsolicited Grant Service (UGS), supporting real-time streams consisting of fixed-size data packets issued at periodic intervals, such as T1/E1 and VoIP without silence suppression;

- Real Time Polling Service (rtPS), supporting real-time data streams consisting of variable-size data packets issued at periodic intervals, such as MPEG video;

- Extended Real Time Polling Service (ertPS), supporting real-time data streams consisting of variablesize data packet issued at periodic intervals, such as VoIP with silence suppression;

- Non Real Time Polling Service (nrtPS), supporting delay-tolerant data streams consisting of variablesize data packets for which a minimum rate is required;

- Best Effort (BE), supporting data streams for which no minimum service level is required.

Such SFs can be created, changed and deleted by DSA, DSC and DSD (Dynamic Service Addition Change - Deletion) signalling, part of the IEEE 802.16 standard. The specification defines three different SF statuses: provisioned, admitted or activated. In the first case the SF is only provisioned via network management system, but resources are not allocated. For admitted SF so-called soft-resources are reserved but data transmission is still not possible. The later is only possible with a SF in active state, which then is associated with a set of assigned resources to be used for carrying traffic data. The mapping between packets and transport SFs is defined by specific classifiers associated with each active SF.

The IEEE 802.16 standard, however, addresses the air interface specification, including the QoS model between BS and SS only. It is the WiMAX Forum [16] which complements IEEE 802.16 with the definition of an end-to-end WiMAX Network Reference Model (NRM), based on an all-IP platform [17, 18]. This architecture includes a whole set of general functional entities required to deliver broadband wireless services. While the WiMAX NRM defines a set of logical components and interfaces, it does not define any specific implementation details but only motivates the application of open standards. It is left to system integrators how to build a complete architecture based on the NRM, either from scratch or based on commercial network components.

The WEIRD architecture, developed by the pan-European research project "EU IST FP6 IP WEIRD (WiMAX Extension to Isolated Research Data networks) [15] and described in this paper, is designed to provide an efficient solution for a WiMAX End-to-End all-IP platform. To the best of our knowledge it is the first WiMAX End-to-End platform built by a research community. The proposed solution is compliant with the WiMAX NRM and to some extent improves existing features and introduces additional functionalities. In particular, it specifies and integrates complete QoS management and control in the IEEE 802.16 radio access network and QoS technologies deployed in the network core. These QoS management and control features of our architecture are in the focus of this paper.

The WEIRD system is specifically designed to some typical WiMAX deployment and application scenarios such as telemedicine and remote (volcano and forest fire) monitoring [2]. Henceforth, the design choices and implementation details presented in this paper render unique and timely insight for any WiMAX system integrator. In order to provide a coherent treatment of the subject we present architectural and implementation details together with performance results of some selected features of the WEIRD QoS framework. 


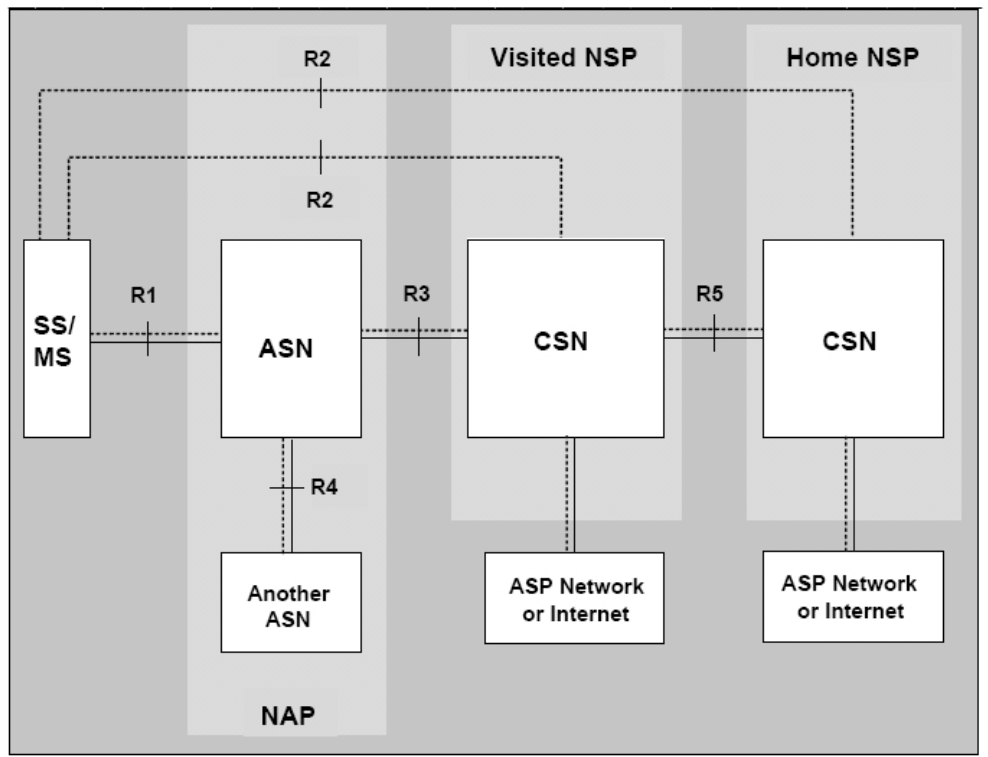

Fig. 1. WiMAX forum network reference model.

The paper is organized as follows: Section 2 shows the WEIRD high level architecture, while Section 3 describes the WEIRD solutions for QoS signalling and resource reservation for different applications. In Section 4 a complete description of the testbed is provided, together with some test results. Finally, Section 5 draws the conclusions.

\section{The WEIRD functional architecture}

The basic policy for WEIRD architecture design was to obey the guidelines for the end-to-end architecture defined by the WiMAX Forum. On top of that we aim to add a set of extensions. This covers, among others, features of the end-to-end QoS control plane, namely QoS signalling, reservation and control functions.

The WiMAX NRM, depicted in Fig. 1, is a logical representation of the all-IP end-to-end network architecture and consists of:

- Subscriber/Mobile Station (SS/MS): a generalized equipment set providing connectivity between subscriber equipment and a BS. The SS/MS may be an host or a CPE supporting multiple hosts.

- Access Service Network (ASN): a set of network functions needed to provide radio access to the WiMAX subscribers.

- Connectivity Service Network (CSN): a set of network functions that provide IP connectivity services to the WiMAX subscribers.

The WEIRD architecture involves all the main entities of the NRM plus some additional components in order to support the before mentioned extensions for QoS management and control. As recommended by NRM guidelines we opted for open standards. For example, the Next Step In Signalling (NSIS) framework $[6,14]$ was chosen for resource reservation signalling along the end-to-end multi-domain path. Therefore, a so-called Connectivity Service Controller (CSC) is placed in each NRM entity 
(SS/MS, ASN and CSN) in order to coordinate and process QoS/NSIS signalling and to perform the resource reservation in the related segment. Another example is the placement of a set of servers and proxies in the CSN providing additional functionalities such as authentication, authorization, monitoring and support for SIP (Session Initiation Protocol) applications. More details on these entities are presented in the sequel of the paper.

\subsection{The WEIRD architecture in compliance with the WiMAX NRM}

The WEIRD architecture follows a multi-plane approach including an Application and Service Layer and a Transport Layer, decoupling high level services from the transport technologies in order to support heterogeneity in core and access network infrastructures. This approach allows the WEIRD system to support a large set of applications for WiMAX relevant scenarios: legacy applications, without signalling capabilities, or applications with signalling capabilities based on different protocols. A specific support is guaranteed for SIP applications with QoS requirements, through the direct interaction between the SIP Proxy located in the CSN and the WEIRD infrastructure. A suitable interface, the WEIRD API, is provided for other signalling applications that can be modified to request resource reservation. Finally, a specific module on the MS, the WEIRD Agent, is adopted to signal QoS requirements for legacy applications. Details for each of these applications are presented in Sections 3.2 and 3.3.

The Transport Layer consists of a Management Plane, a Control Plane, and a Data Plane. The Management Plane includes functionalities for medium and long term resource management, coordinating the static resource pre-provisioning in the Radio Access Network. The Control Plane interacts with application signalling, processing dynamic reservation requests and managing resource allocation in the WiMAX segment and the QoS signalling towards the core network. The Data Plane transports data traffic providing connectivity through different network infrastructures.

The WEIRD architecture, as shown in Fig. 2, includes several modules developed by WEIRD partners (in yellow) integrating protocols such as, Single Network Management Protocol (SNMP) [10] for system management functions and Mobile IP (MIP) [1] (in blue colour).

The main modules included in the SS/MS are:

- WEIRD Agent: provides support for legacy applications to request resource reservation specifying QoS parameters and classifiers.

- Connectivity Service Controller (CSC_MS): handles requests coming from the WEIRD Agent or from signalling applications, performs Admission Control in the MS segment and sends requests to the NSIS module to initiate the end-to-end QoS signalling.

- NSIS Module: manages the NSIS signalling and supports the QoS NSIS Signalling Layer Protocol (NSLP) [5]. It acts as a QoS NSIS Initiator (QNI), the first node in the sequence of the QoS NSIS entities (QNEs) that issue a reservation request for a session.

The main module included in the BS is the WiMAX Resource Controller (WRC) that is configured by the adapter located in the ASN-GW via SNMP protocol and is responsible for the creation and the modification of the Service Flows between the BS and the SS. Service flow configuration is performed by the BS and the MS 802.16 Resource Control modules, through the exchange of MAC messages (DSA, DSC, DSD messages), that are managed directly by the hardware. The BS includes also an SNMP Agent for monitoring. This module allows getting some information about network topology (i.e. MS connected to a specific BS) and bandwidth availability.

The ASN-GW includes the following modules: 


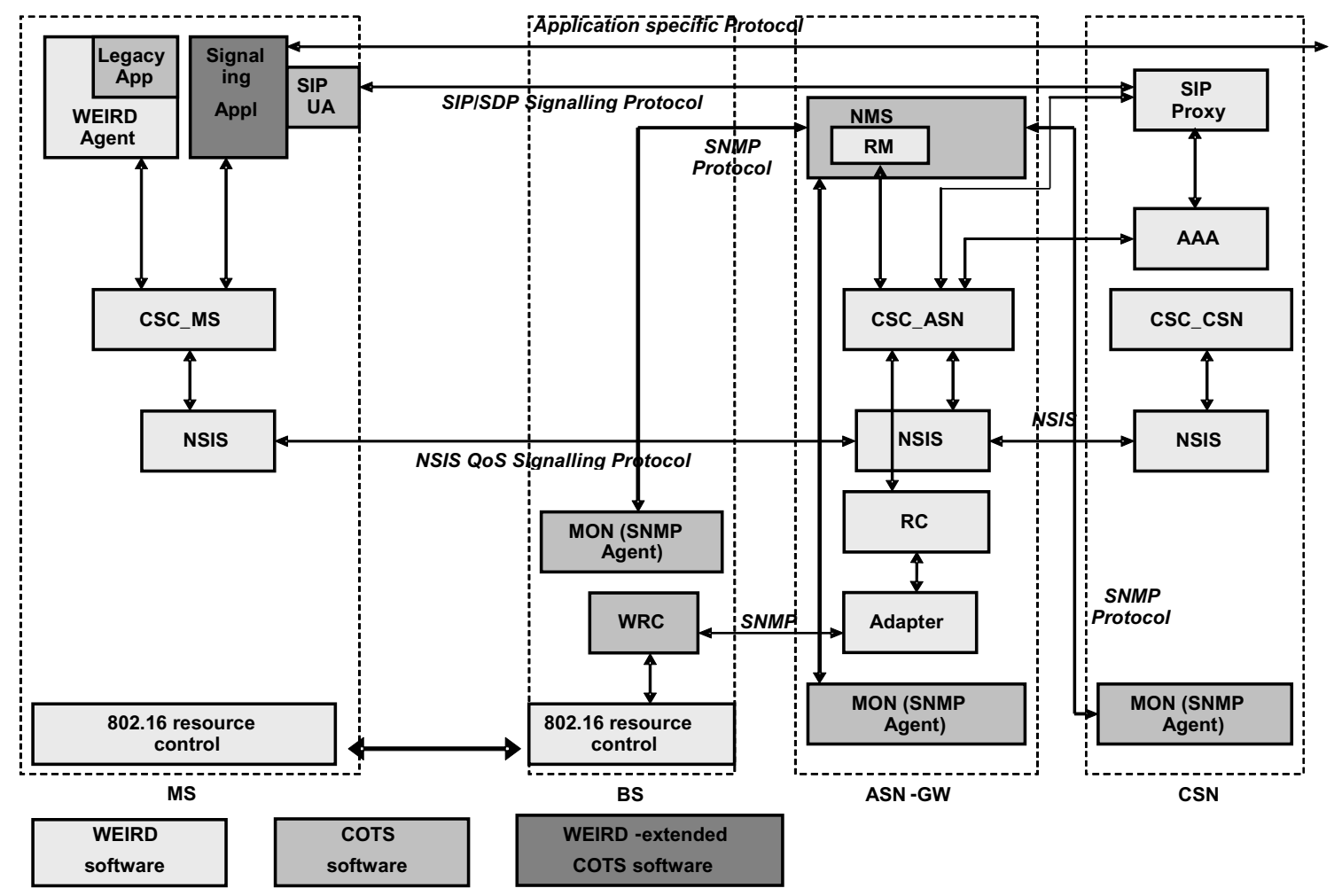

Fig. 2. WEIRD functional architecture.

- Network Management System (NMS), with functionalities for monitoring and network configuration. It includes the Resource Manager (RM) module, used to request the static resource pre-provisioning in the WiMAX segment.

- Connectivity Service Controller (CSC_ASN), the main coordination point for QoS management, resource allocation, Admission Control and QoS authorization. It receives requests from the RM for resource pre-provisioning and from SIP proxy and NSIS module for dynamic resource allocation. The CSC_ASN includes also a database to store information on WiMAX network topology, SF preprovisioning and active service sessions, with a description of resource utilization. Such information can be used to perform Admission Control (AC) in the ASN or to provide monitoring functionalities.

- Resource Controller (RC) manages all the resources in the Radio Access Network (RAN). It receives requests from the CSC_ASN and handles the creation, the modification and the deletion of Service Flows interacting with the adapter.

- Adapter provides an interface to configure the BS and SFs in the wireless link through the SNMP protocol. It adopts the Management Information Base (MIB) defined in the IEEE 802.16f standard [7]. A specific adapter can be provided for each type of hardware in order to support additional proprietary MIBs.

- NSIS module, manages NSIS signalling acting as a generic QNE or a QNI. In the former case it processes requests coming from the NSIS peer and interacts with the CSC ASN that assumes the role of Resource Management Function (RMF). In the latter case it is triggered by the CSC_ASN to initiate a new resource reservation.

The CSN includes the following modules: 
- SIP Proxy, handles SIP signalling, intercepts and processes Session Description Protocol (SDP) messages and interacts with the CSC_ASN to request resource reservation for SIP applications.

- AAA (Authentication, Authorization, and Accounting) Server provides functionalities for user authentication and QoS authorization, interacting with the SIP Proxy and the CSC ASN.

- CSC_CSN, manages resource in the CSN, performing Admission Control in the CSN and coordinating requests coming from the related NSIS module.

- NSIS module, located on the border of the WiMAX domain, acts as an edge QNE. It is in charge of the translation between the parameters of the WiMAX QoS model adopted in the ASN and the specific QoS model adopted in the external domain, see also [3] for details.

The NSIS module in all the entities, SS/MS, ASN and CSN, implements the QoS-NSLP and General Internet Signalling Protocol (GIST) protocols. GIST is responsible for the signalling transport, secure and reliable, while QoS-NSLP deals with the application signalling itself, instructs GIST on the required transport mode (secure, reliable) and encapsulates the QoS specification object (QSPEC). The CSC MS or the CSC_ASN when acting as QNI create the QSPEC objects that contain the details for the desired QoS levels.

The implemented NSIS framework enables legacy applications to request resources, as explained in detail in Section 3.3. These kind of applications do not have native signalling capabilities. These lack of support is compensated by the WEIRD Agent or the WEIRD API (used by customized applications). These modules deal with QoS requests and signalling using NSIS. Similarly, applications with native SIP support leverage the NSIS framework to perform reservations in the CSN-ASN segment and in the MS segment as well as the WEIRD and generic domains.

\subsection{Granted QoS requirements}

The WEIRD architecture provides an efficient integration between the WiMAX network segment and the wired core network through the interoperation of existing signalling mechanisms in order to guarantee end-to-end QoS. The proposed solution offers mechanisms to coordinate general application signalling and SIP signalling with the resource reservation in the WiMAX link and the core network. The basic design requirement to be met was to support the extensive QoS facilities provided by the 802.16 technology end-to-end, such as the specific scheduling services for different kinds of traffic or the one-phase/two-phase SF activation model.

The flexibility of the proposed architecture permits to extend basic QoS provisioning with essential features like an AAA framework based on the Diameter protocol for user and device authentication in order to prevent unauthorized resource utilization. Similarly, the WEIRD end-to-end AC operates in different segments: the SS/MS segment in case of multi-user SS/MS; the ASN segment, including the wireless link and the wired link between the BS and the ASN-GW and finally the CSN segment. Service requests can be admitted or rejected by the AC module accordingly to a user service profile and current network conditions in order to assure QoS guarantees. In summary and greater detail, the WEIRD architecture integrates the following QoS support functionalities:

Static WiMAX resource pre-provisioning. Wireless resources are statically configured between the BSs and the MSs under control of the ASN. SFs with different Scheduling Classes are created to support future traffic flows with different QoS requirements. In this phase SFs are only provisioned and they cannot be used to transfer data.

Dynamic resource allocation as required by legacy applications. The WEIRD Agent signals QoS requirements for new services and requests resource reservation to the CSC $\_$MS. The NSIS module 


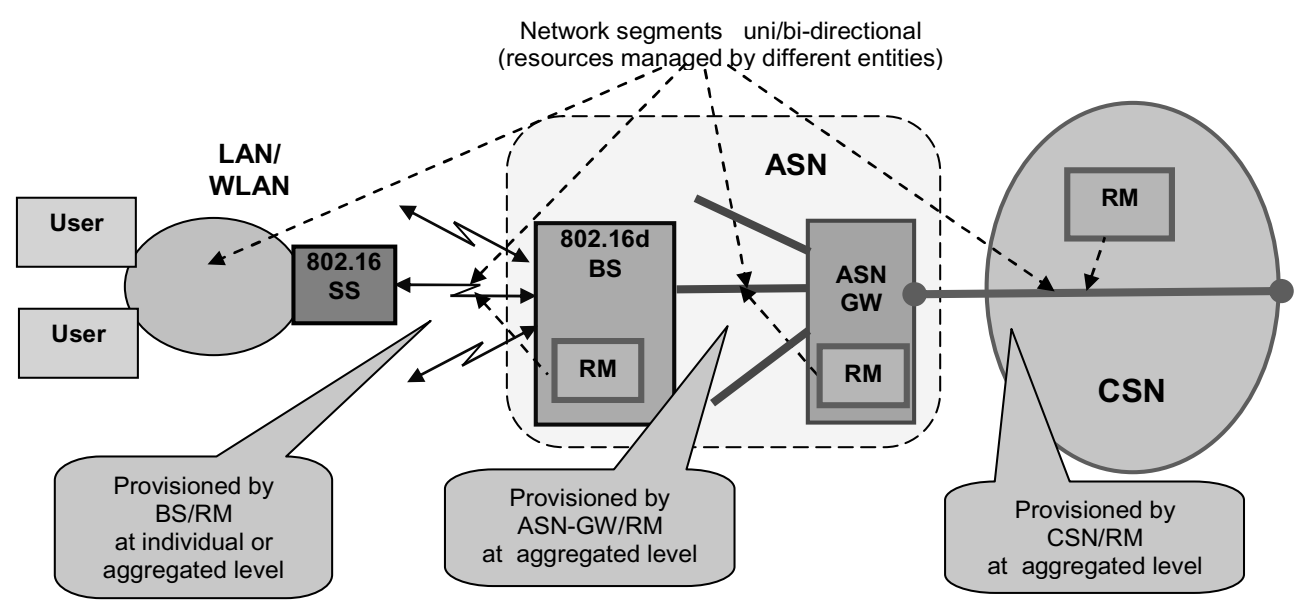

Fig. 3. WEIRD resource provisioning.

located on the MS initiates the end-to-end NSIS signalling towards the receiver. Signalling is intercepted at the ASN-GW and, if the request is authorized and resources are available, suitable SFs in the WiMAX segment are activated.

Dynamic resource allocation as required by SIP applications. SIP signalling coming from the SIP User Agent located on the MS is intercepted and processed by the SIP Proxy of the CSN. The SIP Proxy extracts QoS parameters from SDP messages and interacts with the CSC _ASN that coordinates the activation of the WiMAX SFs adopting the 802.16 two-phase activation model.

QoS authorization. Each request for dynamic resource allocation must be authorized according to the user service profile, through a message exchange between the CSC ASN and the AAA Server.

Admission Control. Each CSC includes an Admission Control (AC) module to allow control of resource reservation and allocation on the respective segment. The Admission Control is based on the current traffic load and the network performance.

Monitoring functionalities are also provided to get information about the WiMAX network topology, the wireless resource utilization and the active application sessions.

\section{WEIRD solutions for QoS signalling and WiMAX resource allocation}

\subsection{Resource provisioning in the Access Service Network}

In order to support QoS in the ASN, appropriate resource provisioning is crucial. Naturally, the challenge of this task is to devise policies and mechanisms tailored to the disparate technologies and entities as well as to take into account the different time scales of resource demand. Furthermore, such provisioning of resources can be static, dynamic, on a per-flow basis or for traffic aggregates. All these features are supported by the WEIRD architecture.

Figure 3, sketches the different segments of the WEIRD ASN. From right to left, an ASN comprises the ASN-GW, a Base Station and a Subscriber Station or Mobile Station. The picture also shows which links are subject of resource provisioning and on what level it is set.

Irrespective of the link technology between two entities, in order to support QoS, the total capacity has to be determined in advance and possibly divided among the supported traffic classes, i.e. supported 
services. In our architecture, the logical module in charge of this task is the Resource Manager (RM), highlighted in green in Fig. 3. This module is a composite of several functions and has a set of interfaces, where the most important ones are an Admin Management Interface (AMI) and the interface to the CSC_ASN. The AMI provides direct access to the RM while the CSC_ASN interface is used for signalling in order to receive resource management orders and to communicate with the Resource Controller (RC), the technology dependent sub-module of the RM.

Both long-term and short-term provisioning can be done by either directly triggering the respective procedure via the AMI or via QoS signalling. In both cases, resource provisioning can be static or dynamic. In the static case, a certain amount of resources required for a class/service is allocated and immediately enabled given that the requestor successfully passes the authentication and authorisation procedure, triggered by the RM after receiving a resource request. This AAA request is communicated back to the CSC_ASN, which in turn forwards the request to the AAA module. Once access is granted, available resources are estimated by the $\mathrm{AC}$ module, and if available, immediately enabled. As the name implies, once enabled, no further changes are possible, except a complete release. This is usually the method for long-term resource pre-provisioning, where the amount of resources is determined by traffic matrix estimation and future prediction. Typically, the inputs for this dimensioning task are commitments contracted between a Network Service Provider (NSP) and its customers, documented in Service Level Agreements (SLA), which cover business relations, and their Service Level Specifications (SLS), which define the technical part.

In contrast to the former feature, dynamic resource provisioning is also supported. The major difference compared to the static case is that resources can be requested and admitted but do not necessarily have to be enabled immediately, instead they can be enabled by a subsequent request. Naturally, the reason for featuring this by our architecture is to support per-flow QoS on the IEEE 802.16 link. As mentioned before, this can be accomplished via the AMI, but the common method anticipated by our architecture is to deploy the inherent NSIS QoS signalling facility or even SIP signalling, as described in the previous and later sections. These requests are handled by the CSC ASN, translated in resource provisioning requests and communicated to the respective RM.

The above elucidated modules and interactions all together belong to the general part of the RM system. What is missing by now is the technology dependent part, which is implemented in the RC. Naturally, resource provisioning for the wired part differs from the wireless part in many aspects. As common practices in today's core networks, resources beyond the BS towards the CSN are statically preprovisioned in our architecture on aggregate level. Basically, the RM is the clearinghouse and does order the $\mathrm{RC}$ to implement the actual resource provisioning. For the wired part, this means the configuration of the typical DiffServ QoS modules, namely policer, classifier, scheduler, etc, as this is the supported QoS architecture.

Just the opposite is the case for the wireless link. Radio resources are scarce and subject to many external influences, like weather or interference from other radio systems. In order to provide QoS given these uncertainties, IEEE 802.16 supports a fine-granular QoS model. Basically, it comes with all the features like described above, dynamic and static, aggregate and per-flow, pre-provisioned and on demand provisioning. Hence, the principle task of the RC is to translate the general primitives into IEEE 802.16 terminology and to invoke the respective hardware calls.

\subsection{Integration of the Session Initiation Protocol}

For native SIP applications the WEIRD approach is based on the integration between pure SIP signalling and mechanisms to allocate resources in the WiMAX network as well as to request resource reservation 


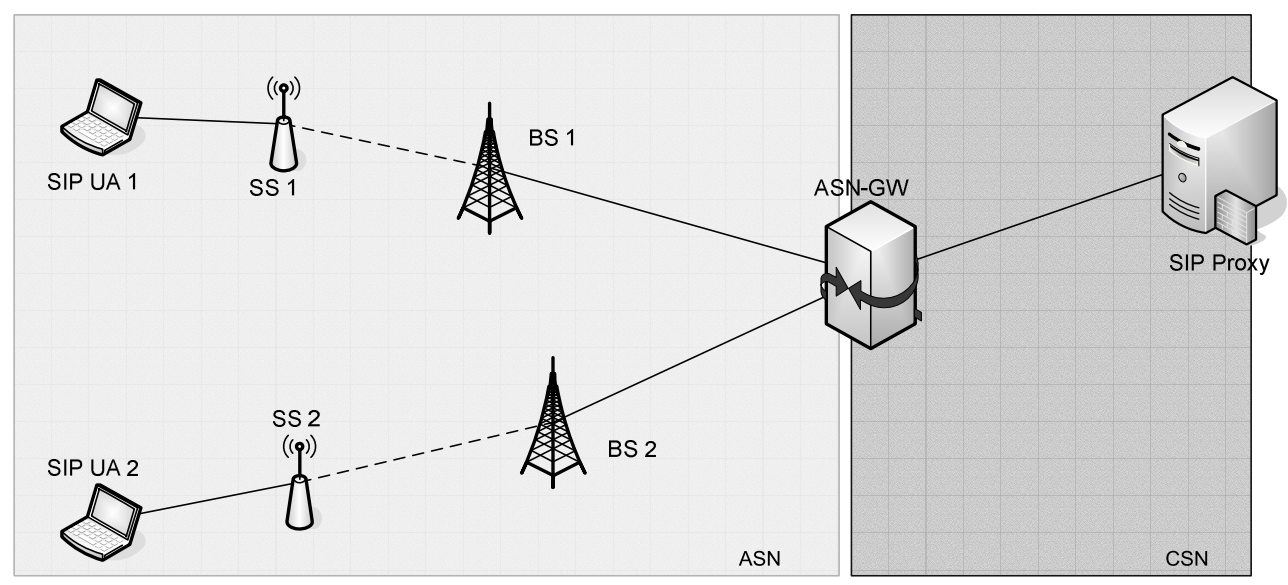

Fig. 4. Scenario with SIP clients connected to the same ASN.

towards the core network. The WiMAX resource allocation includes the creation and the activation of the Service Flows (SFs) for each media flow (audio and/or video) of the SIP call and is handled using a network-initiated approach, where the ASN-GW is the entity in charge of the BS configuration for the SF management. The two-phase activation model as defined in the IEEE 802.16 standard is adopted to activate WiMAX SFs in order to get an efficient bandwidth utilization: during the call establishment each Flow is admitted without any actual usage, and it is activated only when the capability negotiation is concluded.

The resource allocation for a SIP call involving two endpoints located in the same ASN (Fig. 4) requires the creation of uplink and downlink SFs for each media component, while no resources are allocated in the CSN or in the core network. On the other hand, the resource reservation in the core network is required if the SIP clients are located in different ASNs (Fig. 5). In this case, each SIP client is registered by a specific SIP Proxy situated in the CSN connected to the ASN where the client is located. In order to obtain a consistent level of end-to-end QoS along the end-to-end path, resources must be made available not only in the two access networks, but also along the path between the two ASN-GWs. The therefore required QoS signaling interaction is based on the NSIS protocol, in order to support the interoperability among various QoS-enabled domains, characterized by different QoS metrics.

The overall approach for SIP applications is based on the following assumptions:

- SIP signalling is initiated by a standard SIP User Agent (UA) on a host connected to the SS and is intercepted and processed by the Application Function (AF) of the SIP Proxy located in the CSN.

- SDP protocol is adopted to describe multimedia sessions.

- Codec negotiation during session setup can be supported. One participant offers the other a description of the desired session from its perspective and the other participant answers with the desired session from its perspective.

- A QoS Assured model is adopted. The call can be established only if the requested/required QoS specified in SDP messages as attributes of the media can be provided. Therefore, unlike the QoS enabled model, where the resource availability does not affect the success of the SIP call but only the QoS level, in the QoS assured model the resource reservation is a precondition for the SIP call.

- Each SIP method must be authorized through a Diameter message exchange between the SIP Proxy and the related AAA Server. Similarly, each WiMAX resource utilization must be authorized according to the QoS level required for the SIP call and the caller user profile. This QoS authorization is performed through a Diameter message exchange between the ASN-GW and the AAA Server. 


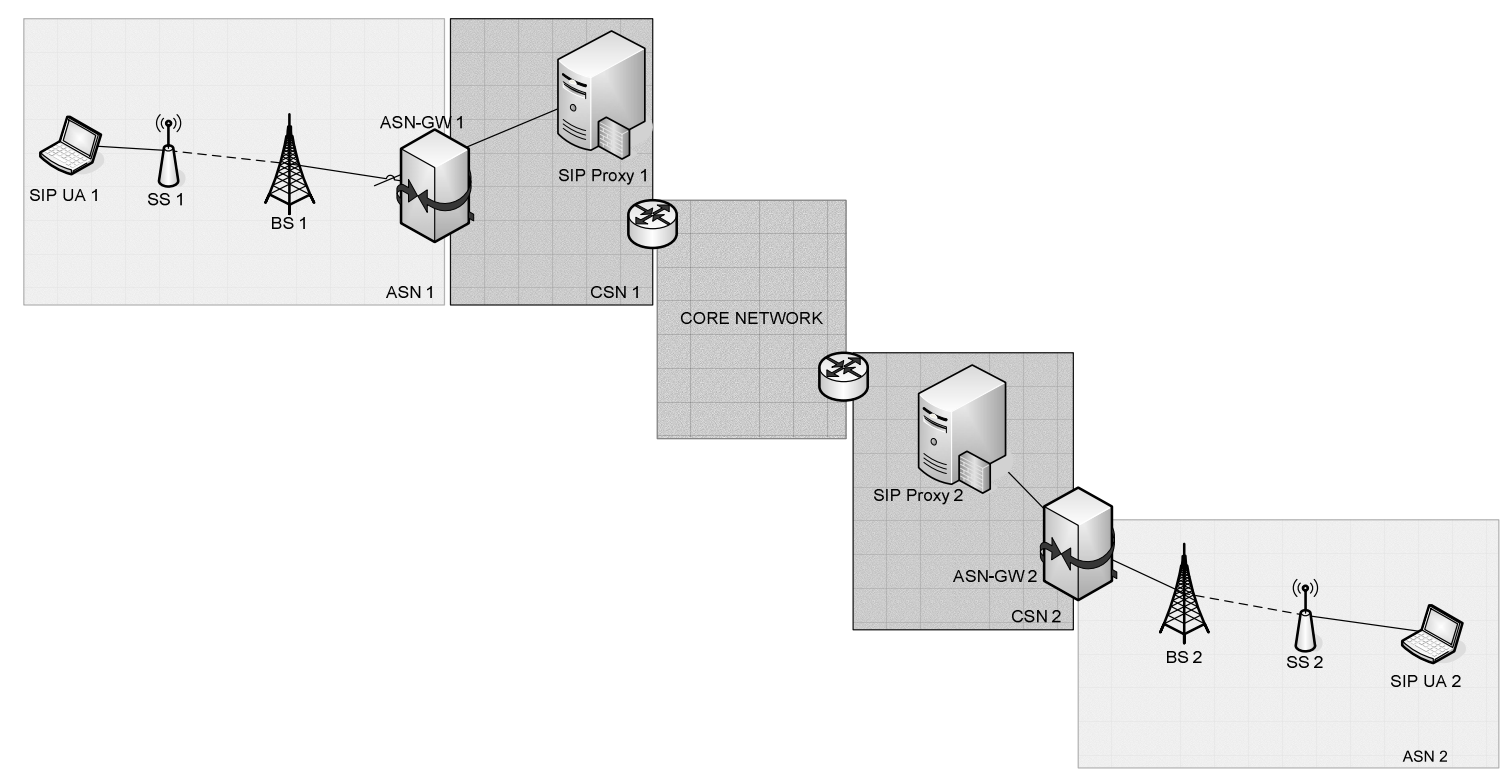

Fig. 5. Scenario with SIP clients connected to different access networks.

The signalling process can be considered as a two-phase process. In the first phase the calling proxy extracts the max bandwidth from the SDP payload and requests resource reservation for the worst-case (max bandwidth) to the related CSC_ASN. In this phase resources in the WiMAX network are only admitted and cannot be used to carry traffic data, recall the IEEE 802.16 QoS model described in Section 1. In the second phase, both proxies modify or confirm bandwidth requirements after the codec negotiation phase is concluded and WiMAX SFs are activated.

The interface between the SIP Proxy and the CSC_ASN is based on the Diameter Gq' interface [3, 13]. Two types of Diameter messages are supported: the AAR/AAA (Authorize Authenticate Request/Answer) to request the resource reservation and the STR/STA (Session Termination Request/ Answer) to signal the termination of the service session.

The interface between the SIP Proxy and the AAA Server is based on the ETSI/TISPAN Cx/Dx interface and involves the MAR/MAA (Multimedia Auth Request/Answer) Diameter messages for the authentication and the user authorization. Each SIP method is authenticated using the HTTP Digest Authentication.

The interface between the ASN-GW and the AAA Server is based on the Diameter Quality of Service Application [13]. The QoS authorization is performed through the exchange of QAR/QAA (QoS Authorization Request/Answer) Diameter messages that include specific AVPs (Attribute Value Pairs) for the description of the required QoS.

Figure 6 shows the SIP message exchange between SIP UAs and SIP Proxies, and the Diameter Gq' message exchange between SIP proxy and CSC ASN. The caller SIP UA is located on the SS/MS1, included in the Radio Access Network under control of the ASN_1. The SIP Proxy for the caller SIP UA is located in the CSN_1 and includes the AF1. The SIP UA of the called party is located on the SS/MS2 and is served by the CSN 2 SIP Proxy that includes the AF2.

When the AF1 receives the first SIP INVITE, the media flow description is extracted from SDP messages and a Diameter AAR message is sent to the CSC ASN1, requesting resource reservation for the max bandwidth. The CSC_ASN1 performs QoS Authorization interacting with the AAA Server and 


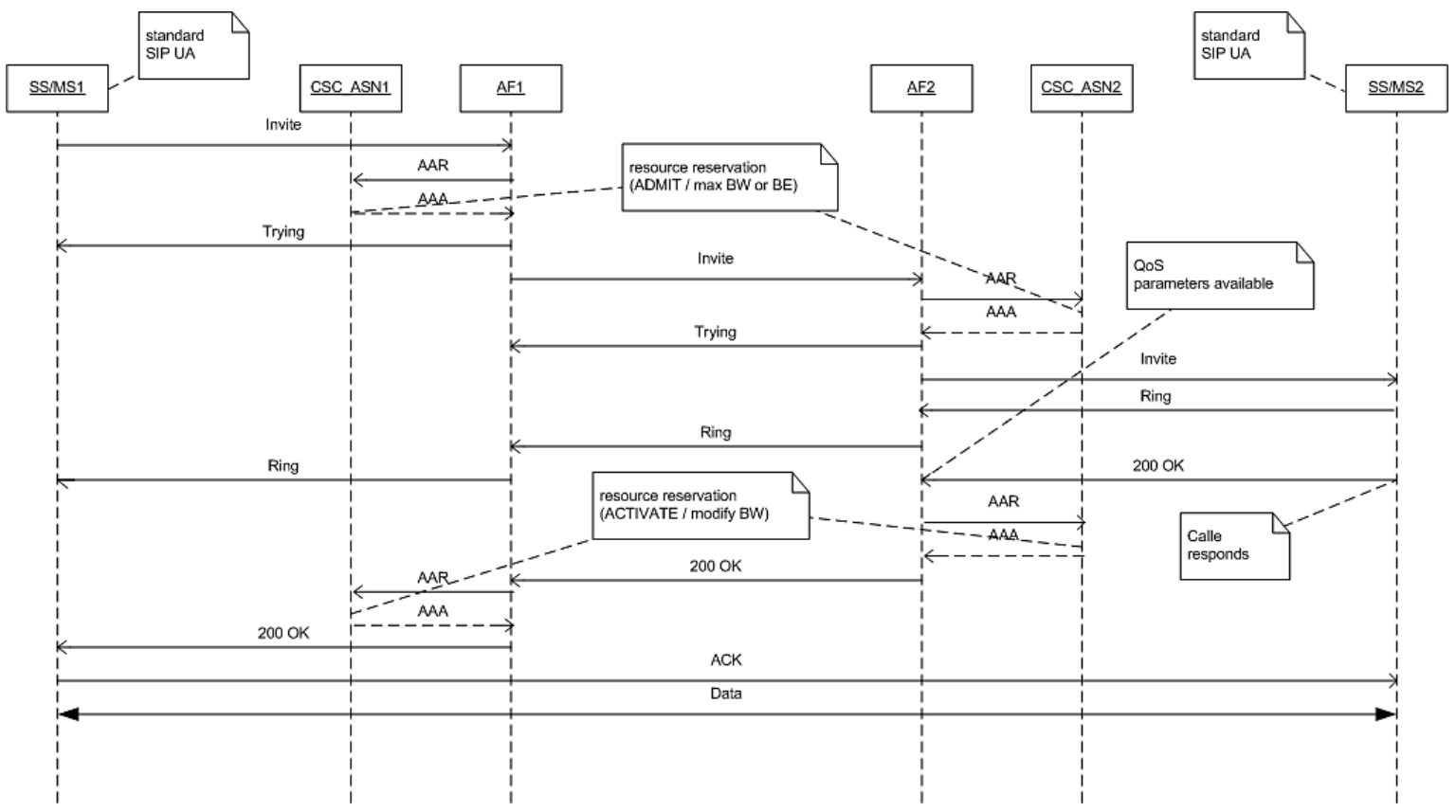

Fig. 6. Interaction between SIP signalling and WiMAX resource allocation.

Table 1

Mapping between media type and WiMAX scheduling class

\begin{tabular}{cc}
\hline Media type & Scheduling service \\
\hline AUDIO & UGS \\
VIDEO & rtPS/ertPS \\
DATA & nrtPS \\
TEXT & BE \\
\hline
\end{tabular}

AC on the WiMAX segment and verifies resource availability in the core sending an NSIS query. If resources are available in each segment, an uplink SF and a downlink SF are created and admitted, using the Resource Controller and the Adapter. The scheduling class associated to each SF depends on the type of the related media component, as specified in the SDP message. The mapping between the media type and the WiMAX scheduling service is shown in Table 1. The admission result is communicated to the AF1 with the Diameter AAA message. The SIP INVITE is forwarded and intercepted by the SIP Proxy 2. SFs are admitted in the Radio Access Network 2 using a similar approach.

When the called party responds, the SIP UA 2 sends a SIP 200 OK message including the negotiated bandwidth. This value is placed in the media flow description of the new AAR messages. In this phase the two CSC_ASNs activate the related WiMAX SFs and send a request for resource allocation in the core network using a NSIS Reserve message.

\subsection{QoS signalling for legacy applications}

The WEIRD architecture is not limited to applications with native SIP or NSIS modules but aims at supporting a large number of different applications. Such applications can be categorised into two 


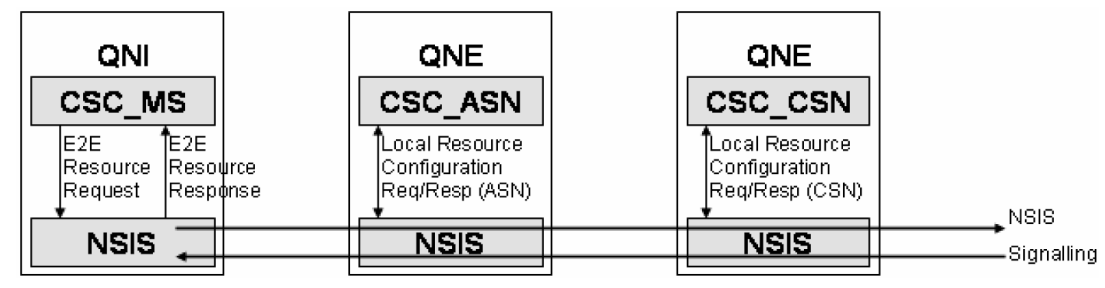

Fig. 7. NSIS Signaling for legacy applications.

distinct groups: applications which allow customization, i.e. open source software, and their counterpart. We call it closed source software.

To support the first type of applications the WEIRD consortium defined the WEIRD API (WAPI). This API provides a set of functions among which QoS signalling is one example. In brief, the API provides an interface to the complete NSIS framework. To enhance customizable applications with QoS features given this API is rather straightforward. Some of the details are described in the sequel.

So called legacy or closed-source applications cannot be modified. In order to support these applications too the WEIRD Agent has been developed. This software module basically serves as translator. It is an off-line pre-provisioning module which configures the channel right before the application enters operational mode. This module is preconfigured with application specific QoS requirements and classifiers (IP addresses, port numbers, protocol, TOS (Type of Service) code) and triggers the resource allocation interaction with the CSC_MS through the interface provided by the WEIRD API. The QoS request is processed by the CSC_MS that performs AC in the MS/SS segment in case of multi-user (multi-application) SS/MS and eventually triggers the NSIS module to initiate end-to-end resource reservation. In this scenario the CSC_MS acts as a QNI and is the main entity that handles and coordinates QoS signalling. Since SF management obeys an network-initiated approach the MS/SS QNI is not actively involved in WiMAX resource allocation but only handles end-to-end signalling. In particular, the QNI creates the messages for the allocation, the modification and release of resources required by the application sessions through the creation of RESERVE, UPDATE and TEAR-DOWN QoS NSLP messages as defined in [5].

Individual application's QoS parameters and classifiers are encapsulated in the QSPEC object while user credentials are part of the AUTH_SESSION object, defined in [11]. These values are extracted at the ASN-GW and processed by the CSC_ASN which subsequently performs QoS authorization, AC and in case of success, resource allocation in the WiMAX segment. The CSC ASN is the Resource Management Function (RMF) in charge of ASN configuration for both the wireless and the wired link (between the BS and the ASN-GW). In case of successful resource reservation the QoS signalling messages are forwarded and processed along the path. The NSIS messages are similarly processed by all nodes in the signaling path and which are located in the CSN and the core network as shown in Fig. 7.

It should be noted that while both, the NSIS nodes on the SS/MS and the ASN-GW are part of WiMAX domain (RAN), other NSIS nodes toward the destination may be part of different QoS domains, like DiffServ or IntServ clouds. The resource configuration in each segment is therefore to be based on the respective QoS model. Interoperability between different QoS models is a well known issue and assured by mechanisms defined by the NSIS protocol suit. Commonly a mapping between different types of QSPEC objects, one for each QoS model, is performed by domain edge nodes. For example, the initiator QSPEC created by the MS/SS QNI reflects the WiMAX QoS model and includes all the QoS parameters required for the configuration of WiMAX SFs. Beyond the WiMAX segment, the initiator QSPEC must be mapped to local QSPECs and be placed on the top of the QSPEC stack such that internal nodes process 


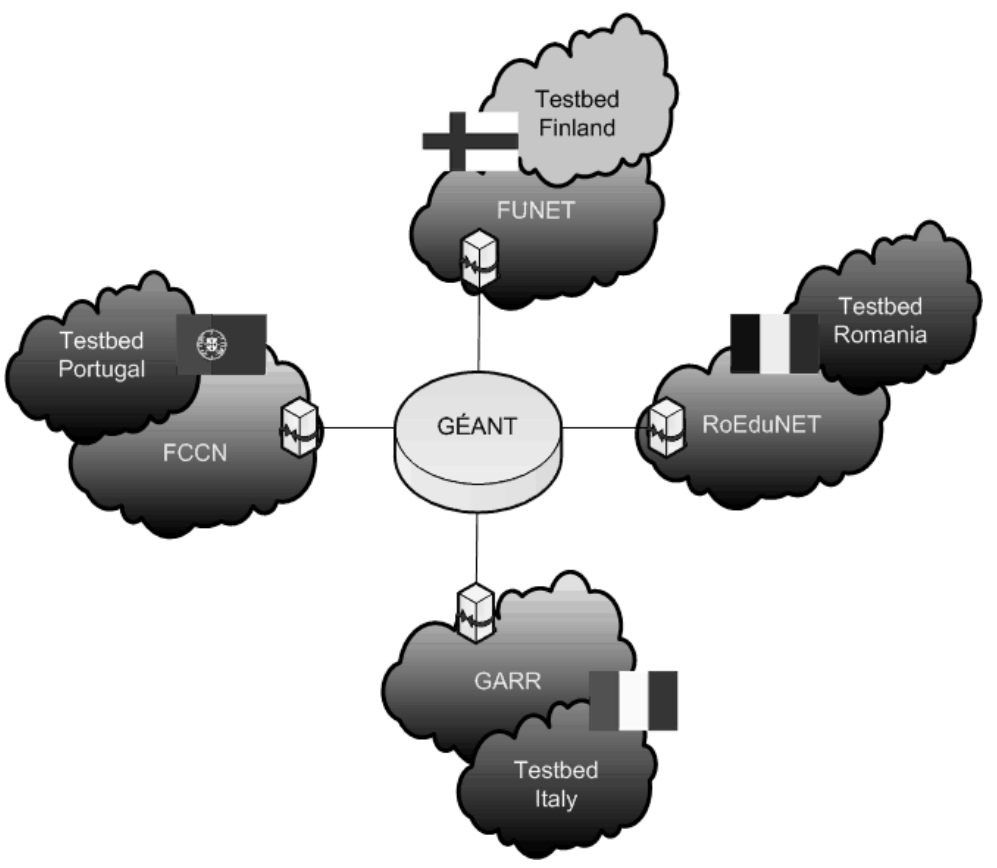

Fig. 8. WEIRD project testbeds interconnected through GEANT research network.

only the local QoS parameters, omitting the attributes that otherwise could not be understood anyways. A more detailed treatment of this subject can be found in [12].

\section{Testbed implementation and functional testing}

\subsection{WEIRD demonstration testbed}

The technical solutions developed within the WEIRD project and described in this paper have been implemented and tested in four European testbeds deployed in Finland, Portugal, Italy and Romania. These national clouds are interconnected by the European research network - GEANT. For a high-level view see Fig. 8.

In order to evaluate system performance and utility, several scenarios that can take advantage from both, the WiMAX technology and the architecture foreseen by the WEIRD project have been defined, mainly one for each testbed [4]. Specifically, e-health, fire-prevention, video-streaming and environmental monitoring have been the core scenarios defined by WEIRD. Although WiMAX is installed and common to all testbeds, each testbed has different profiles and technologies according to the selected scenario. Thus, one of the key features of the WEIRD framework is its support for heterogeneity, inherently reflected in the testbeds, with respect to the implemented application scenarios.

For general testing purposes a demonstrator, as illustrated in Fig. 9, comprising the Italian and the Finnish testbed, interconnected by GEANT was installed. It is based on commercial WiMAX certified equipment and completed by the WEIRD architecture. The Finnish testbed is equipped with a Video on Demand (VoD) server and serves as origin for video content distribution over GEANT to a VoD client in Italy, which is connected to the network via a WiMAX SS. 


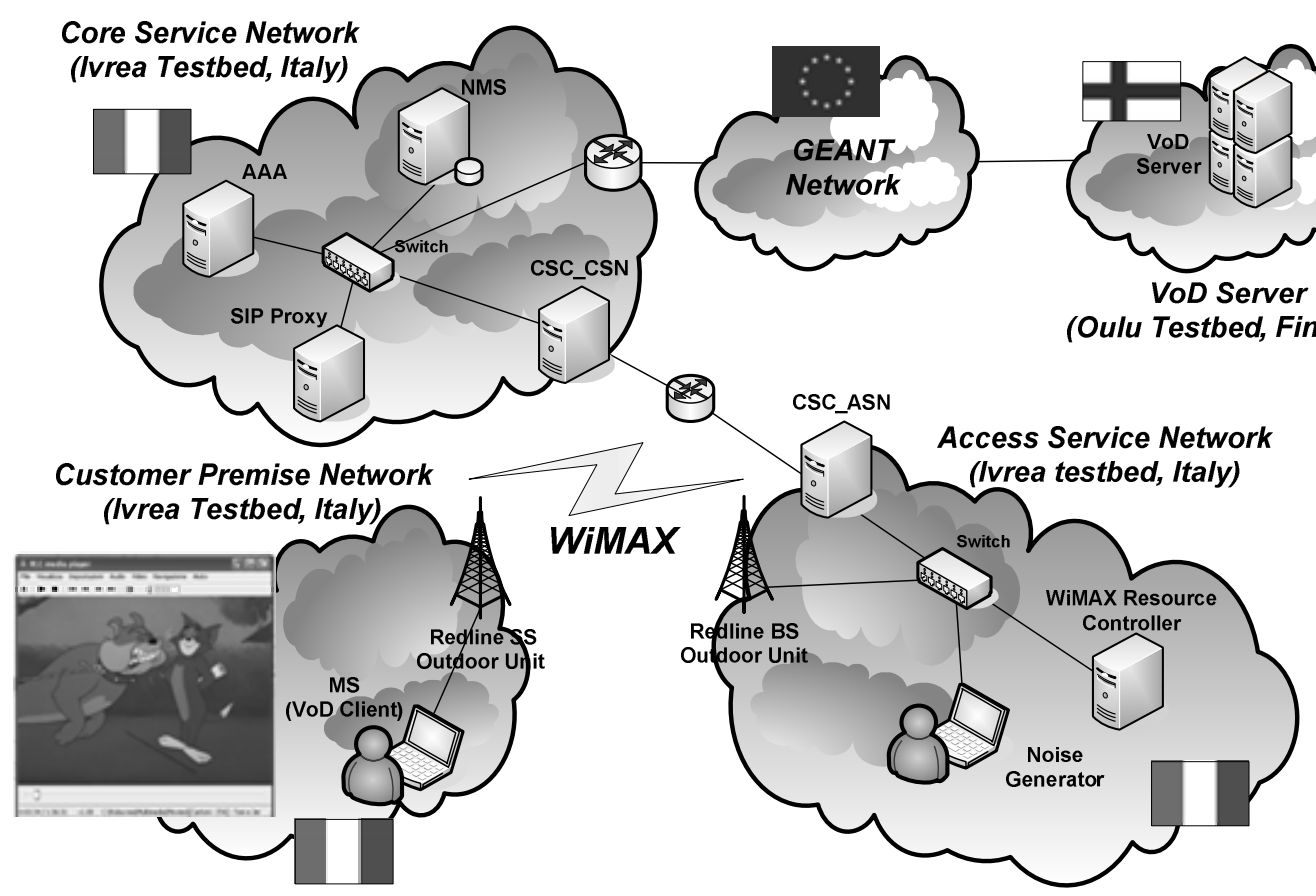

Fig. 9. WEIRD demonstration testbed.

The CSN of either the Finish or Italian site is connected to the GEANT edge router. It implements all the modules presented in previous sections, namely the CSC_CSN, NMS, AAA and SIP Proxy. Moreover, the CSN is responsible for establishing connectivity with the IP backbone and features a DHCP and DNS server.

As defined by the WiMAX Forum NRM, see Fig. 1, each site CSN is connected to an ASN. The latter is responsible to perform relay functions to the CSN in order to establish IP connectivity and AAA mechanisms. At the network edge a single SS serves as WiMAX based Radio Access Network (RAN). A MS is directly connected to the SS resembling a typical "last mile" scenario. Finally, the VoD client runs on the MS as shown in Fig. 9.

\subsection{Functional and performance evaluation}

After one and a half year of design, system development and integration, this work presents the sum of the very first test results. Naturally, such initial tests conducted in the context of such a complex system mainly focus on functional testing first. As central to our work, we focus on the validation of the proposed QoS management and control architecture described in earlier sections. Recall that the implementation of these features has not been specified in any standard. Therefore, our framework presents a unique solution to this problem at real network scale.

The performance tests were conducted between the MS and the CSN acting as a VoD Server. Data and signaling paths (path-coupled mode) include all NSIS aware nodes from the origin to the destination in each network segment, except on GEANT network which is a transparent cloud. The WEIRD Agent module installed at the MS enables the creation of downlink and uplink reservations for the traffic originated at the VoD server and at the VoD client, respectively. Each test accommodates a specific number of reservations to simulate different clients, thus the available bandwidth is different as well. 
Table 2

Number of reservations vs. bandwidth used in tests

\begin{tabular}{ccc}
\hline Number of reservations & Downlink bandwidth & Uplink bandwidth \\
\hline 2 & $9 \mathrm{Mbps}$ & $9 \mathrm{Mbps}$ \\
32 & $36.6 \mathrm{Kbps}$ & $36.6 \mathrm{Kbps}$ \\
64 & $18.3 \mathrm{Kbps}$ & $18.3 \mathrm{Kbps}$ \\
256 & $4 \mathrm{Kbps}$ & $4 \mathrm{Kbps}$ \\
\hline
\end{tabular}

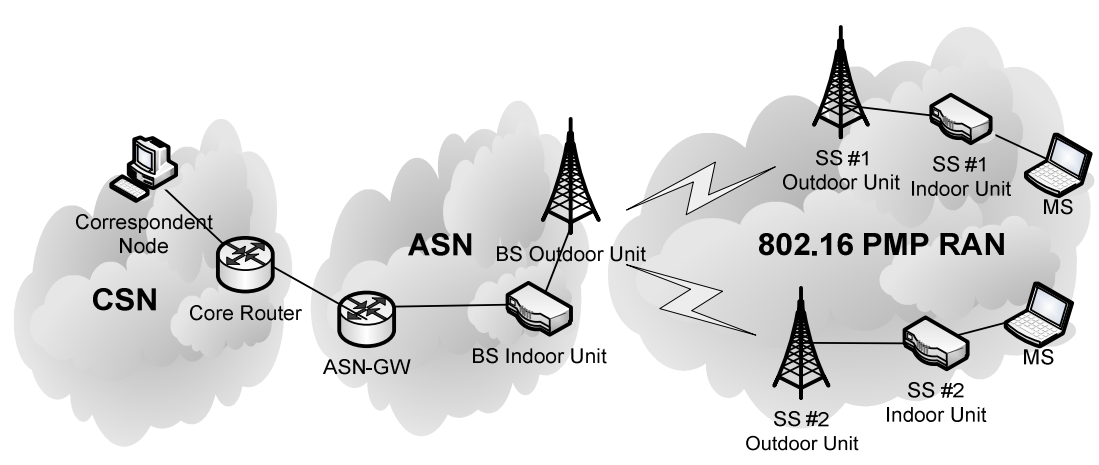

Fig. 10. QoS framework testbed.

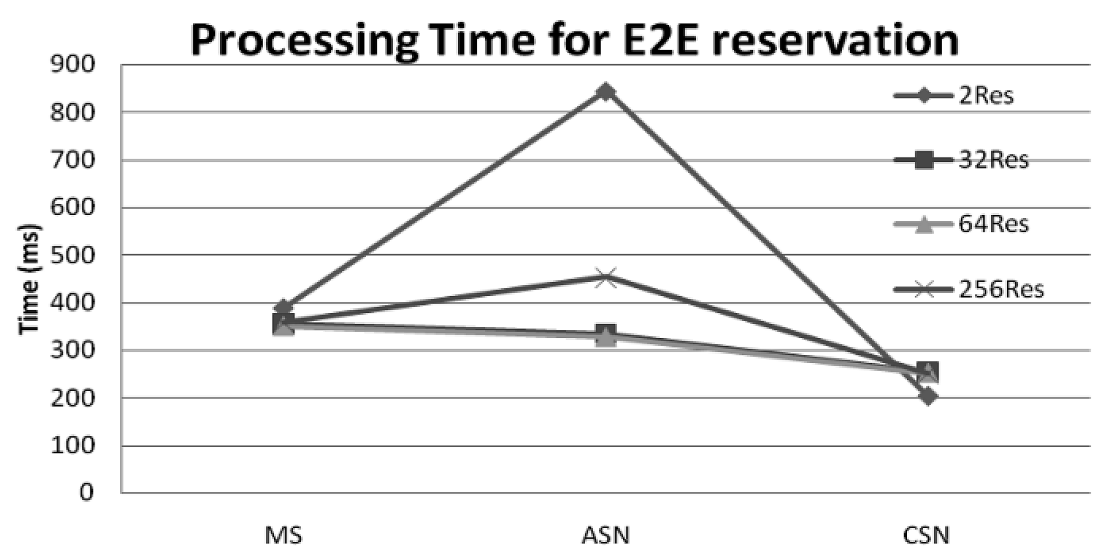

Fig. 11. E2E chain processing time.

Table 2 summarizes the relation between the number of reservations and the bandwidth parameter of each test.

In order to better understand the modules performance of our architecture, the evaluation considered different protocols and parameters. For instance, NSIS modules are split between its two components: GIST, the generic transport layer, and QoS NSLP, the QoS signaling layer, includes message processing and internal processing times. The CSCs include QSPEC serialization/deserialization, message mapping, serialization of Message Routing Information (MRI) objects, as well as admission control and policy functions. The RC and adapter modules include all the processing associated to the creation and deletion of services flows in the WiMAX equipment. Figure 12 presents the performance of each software component, namely GIST, QoS NSLP, CSCs, RC and Adapter plus WiMAX. Measurements indicate that the processing overheads introduced by the CSCs modules have the highest impact in the reservation process. In the 32 reservations case, for instance, the accumulated processing time of 


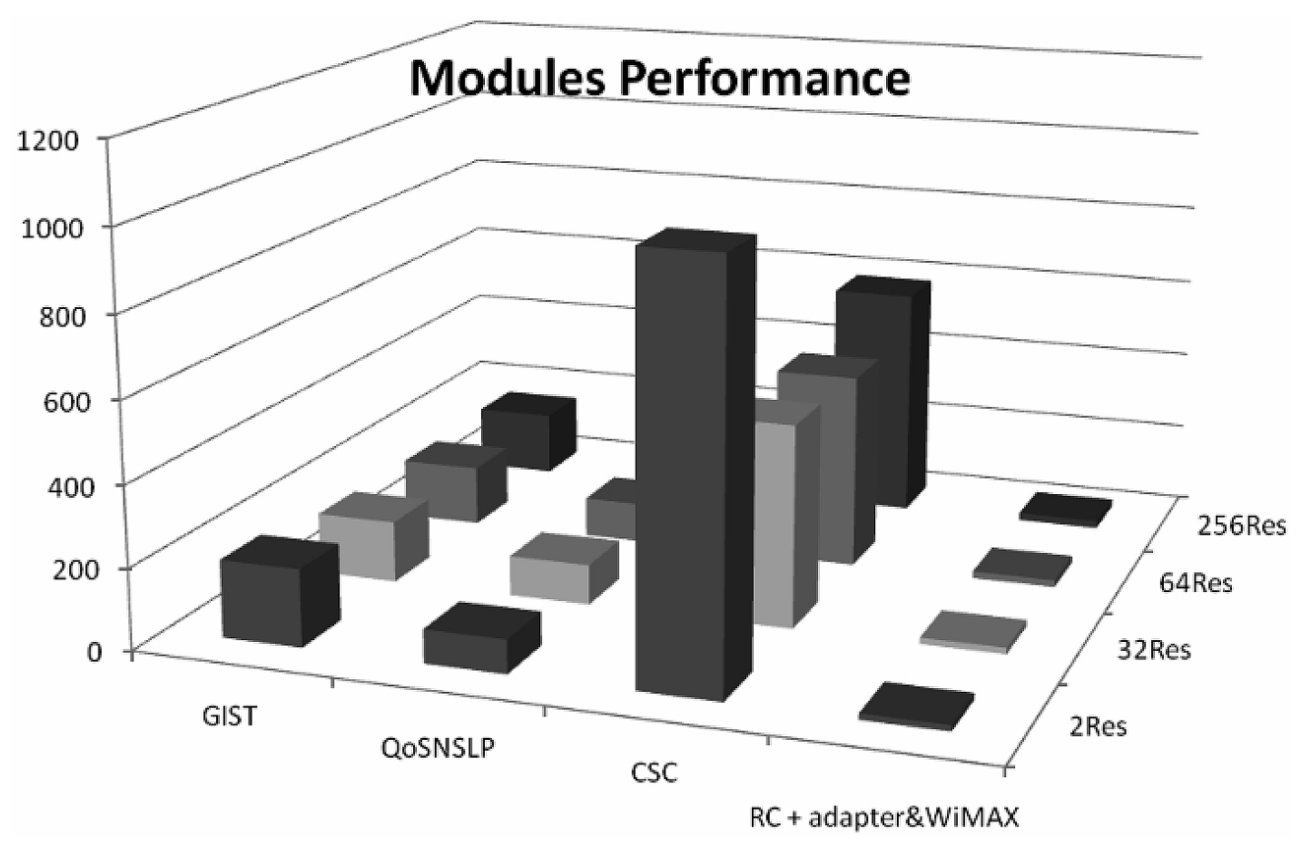

Fig. 12. Software modules performance (ms).

CSC_MS, CSC_ASN and CSC_CSN is around 500ms. Also to remark that the cases with 2 reservations have the highest processing time due to the session setup in the different modules and initial processes such as NSIS transport layer message association. With the increased number of reservations the processing time of the different modules does not increment significantly. For instance, in the 64 reservations case the accumulated processing time of CSC_MS, CSC_ASN and CSC_CSN is around 510ms while with 256 reservations it is around $580 \mathrm{~ms}$.

We explain these performance differences by different programming languages and, on top of that, individual specifics of each module. For example, the difference between the best performing module (RC) and the worst (CSCs) can be explained by their great semantical difference.

Besides the contribution of each module in the overall signaling chain, the performance of each entity, namely the MS, the ASN and the CSN is also a matter of study. Figure 11 presents the processing time for the end-to-end reservation chain, split between the MS, the ASN and the CSN. The ASN is the major contributor for the delay in the overall chain, since it is responsible for radio connectivity functionalities and relaying to the CSN. The MS is the second entity to present more delay since it is responsible for the QoS initiation parameters, such as serialization of QSPEC and MRI objects used by NSIS protocols. The different test cases have the same performance values with 32 and 64 reservations, with values lying around $340 \mathrm{~ms}$ in the ASN. The worst case occurs with 2 reservations, presenting a processing time around $844 \mathrm{~ms}$; such high value is justified with the session initiation of the different modules and message association of NSIS protocols. When there are 256 reservations, the processing time in the ASN also increases, simply as it has to perform a large number of functions related with the CSC_ASN, some of them presented in previous sections.

Without having a reference benchmark we can only base our conclusions on experience and intuition. Nevertheless, we believe that the end-to-end processing overhead introduced by the QoS signaling integrated in the WEIRD system is within acceptable bounds. Especially if there are multiple requests 
able to reuse NSIS associations. In such a situation the measured initial setup times around 800ms appear like a reasonable price to pay for the level of QoS which is then provided for the complete flow lifetime.

\section{Concluding remarks}

This paper presents an architectural solution for a complete WiMAX end-to-end network based on the recommendations and guidelines of the WiMAX Forum NRM while taking into account WiMAX specific application and deployment requirements.

Given the scope of the complete WEIRD system the focus of this work is on a specific feature set, namely QoS provisioning comprising resource management, resource request signalling and reservation as well as resource control, for which we have presented architectural design details.

In this context the proposed architectural solution exhibits adequate flexibility by supporting a complete range of applications with respect to signalling. In detail these applications are:

- SIP applications: direct interaction between SIP Proxy and ASN-GW.

- Open-source applications: a particular API (WAPI) is provided in order to interface with the WEIRD QoS signalling framework.

- Closed-source applications: A WEIRD Agent is used as translator between the applications and the WEIRD system.

As we are concerned with the design, implementation and integration of a real system, the evaluation of our architecture was more focused on functional testing than its performance. For the selected scenario we could show that the end-to-end QoS signalling and resource reservation interaction performed according to our design and revealed acceptable performance figures.

Another valuable result of our work was the confirmation that developed framework operated over several domains and with disparate hardware components by different vendors. The proposed structure allows exploiting individual characteristics without a significant lack of generality. Hence, our work can be understood as a confirmation of the WiMAX Forum NRM guidelines.

\section{Disclaimer}

The work described in this paper is based on result of IST FP6 Integrated Project WEIRD. WEIRD receives research funding from the European Community's Sixth Framework Programme. Apart from this, the European Commission has no responsibility for the content of this paper. The information in this document is provided as is and no guarantee or warranty is given that the information is fit for any particular purpose. The user thereof uses the information at its sole risk and liability.

\section{References}

[1] C. Perkins, IP Mobility for IPv4, IETF RFC 3344, 2002.

[2] E. Angori et al., WiMAX Applications to Extend National Research and Education Networks, ANIPLA'06, Rome, Italy, 2006.

[3] F. Alfano et al., Diameter Quality of Service Application, IETF Internet Draft, 2006.

[4] F. Andreotti et al., WEIRD Testbeds with Fixed and Mobile WiMAX Technology for User Applications, telemedicine and monitoring of impervious areas, TRIDENTCOM 2008.

[5] G. Ash et al., QoS NSLP QSPEC Template, IETF Internet Draft, 2006. 
[6] H. Schulzrinne et al., GIST: General Internet Signalling Transport, IETF Internet Draft, 2007.

[7] IEEE Standard for Local and Metropolitan Area Networks. Part 16: Air Interface for Fixed Broadband Wireless Access Systems. Amendment 1: Management Information Base, IEEE Std 802.16f, 2005.

[8] IEEE Standard for Local and Metropolitan Area Networks. Part 16: Air Interface for Fixed Broadband Wireless Access Systems, Amendment 2: Physical and Medium Access Control Layer for Combined Fixed and Mobile Operation in Licensed Bands, IEEE P802.16e/D12, 2005.

[9] IEEE Standard for Local and Metropolitan Area Networks. Part 16: Air Interface for Fixed Broadband Wireless Access Systems, IEEE STD 802.16 2004.

[10] J. Case et al., A Simple Network Management Protocol (SNMP), IETF RFC 1157, 1990.

[11] J. Manner et al., Authorization for NSIS signalling Layer Protocol, IETF Internet Draft, 2006.

[12] N.Ciulli et al, A QoS model based on NSIS signalling applied to IEEE 802.16 networks, 2nd IEEE Workshop on Broadband Wireless Access, Las Vegas, USA, 2008.

[13] P. Calhoun et al., Diameter Base Protocol, IETF RFC 3588, 2003.

[14] R. Hancock et al., Next Step in Signalling (NSIS): Framework, IETF RFC 4080, 2005.

[15] WEIRD project web site, http://www.ist-weird.eu.

[16] WiMAX Forum web page, www.wimaxforum.com.

[17] WiMAX Forum, WiMAX End-to-End Network System Architecture, Stage 2: Architecture Tenets, Reference Model and Reference Points, 2006.

[18] WiMAX Forum, WiMAX End-to-End Network System Architecture, Stage 3: Detailed Protocols and Procedures, 2006.

Thomas Michael Bohnert received his Dipl. Ing. degree in Computer Engineering from the University of Applied Sciences Mannheim, Mannheim, Germany in 2005. Thereafter he joined the University of Coimbra, Portugal from which he is expected to receive his PhD in Fall 2008. After visiting Tampere University of Technology and VTT Technical Research Centre of Finland in Finland, and Beijing University of Posts and Telecommunications in China, between end 2006 and end of 2007, he joined Siemens Corporate Technology in January 2008. His research interests range from QoS and QoE control in wired and wireless networks, in particular WiMAX QoS/QoE, over Carrier Grade Ethernet Services, Future Internet Architectures to subjective VoIP Quality Assessment. He is founder and general chair of the IEEE Broadband Wireless Access Workshop (www.bwaws.org) and is involved in various European and German projects like IST FP6 IP WEIRD, CELTIC/EURECA 100Get or ESF COST290. For the latter he serves as chair of the WiMAX Special Interest Group. Additionally he is involved in several international conferences like IEEE ICC, IEEE GLOBECOM, IEEE CCNC, WWIC, NEW2AN and CHINACOM. $\mathrm{He}$ is also Guest Editor for Elsevier Computer Communications and EURASIP Wireless Communications.

Marco Castrucci graduated in Telecommunication Engineering with 110/110 cum laude in May 2006 at the University of Rome "La Sapienza". At present he is pursuing a Ph.D. in Systems Engineering at Computer and Systems Science department of University of Rome "Sapienza". His main research activities concern the design of network architectures for the integration of heterogeneous (both wired and wireless) telecommunication technologies, and the design and simulation of algorithms for Quality of Service (Connection Admission Control) and routing (path selection, load balancing). He participated in the EU FP6 WEIRD project and, at present, he is participating in two EU FP7 projects (OMEGA, MICIE).

Nicola Ciulli, responsible for the Networking Area of Nextworks, received the Laurea degree "cum laude" in Telecommunication Engineering in 1997 at the University of Pisa, and the Degree Diploma at SSSUP S. Anna. His main research activities concern the architectural aspects of Control and Management Planes and of Traffic Control in next generation packet networks: Quality of Service (QoS) provision over the Internet and its interworking with Asynchronous Transfer Mode (ATM), Differentiated Services (DiffServ), MPLS and their integration. A special focus of his research and industrial activity is on GMPLS control and management planes architectures for SDH networks.

He participated in several ACTS and FP5/FP6 IST projects (MAESTRO, PETERPAN, BASS, MOICANE, WEIRD, PHOSPHORUS), in research activities funded by the Italian Ministry for Research and Education (MIUR), and in industrial projects (e.g. Marconi ASTN/GMPLS project, where he coordinated the activities of Nextworks, Alcatel projects on 4G mixed packet/TDM switches).

Giada Landi received the Laurea degree "cum laude" in Telecommunication Engineering at the University of Pisa, Italy, in 2005. Her main research activities concern the design, development and integration of Control Plane in network architectures supporting WiMAX technologies, with main focus on Quality of Service, Admission Control and mobility. She partecipated in the IST FP6 WEIRD project and in research activities funded by the Italian Ministry for Research and Education.

Ilaria Marchetti was born in Italy on June 11th 1978. She received the "Laurea" degree (M.Sc.) with full marks in Electronic Engineering from the University of Rome "La Sapienza", Italy, in 2003. She obtained the Ph.D in Systems Engineering at the 
DIS Department (November 2003 - October 2006) of the same University. She has been a Research Assistant in Control theory and network management at the University of Rome "La Sapienza" (2004-2007) and she is presently a Research contractor. In the framework of her activity, she has mainly researched on the application of control theory on the management of shared resourced. In particular on the application of optimal and predictive control on the framework of QoS/resource management procedures for the third and forth generation of mobile systems.

Cristina Nardini was born in Italy on August 26th 1981. She obtained her B.S. and M.S. degrees in Computer Science from the University of Rome "La Sapienza", Italy, in 2005 and 2007 respectively She participated in research and development activities for the IST EU WEIRD project. At the moment, she is working as Technology Consultant at Hewlett Packard in Telecommunication field.

Bruno Sousa obtained master degree at the University of Coimbra in 2008. He participated in research and development activities for the IST EU WEIRD project. At the moment, he is enrolled as a PhD student on the same University, performing research in the Telecommunication and Telematics group.

Pedro Neves received his B.S. and M.S. degrees in Electronics and Telecommunications Engineering from the University of Aveiro, Portugal, in 2003 and 2006 respectively. From 2003 to 2006 he joined the Telecommunications Institute (IT), Aveiro, Portugal, and participated in the DAIDALOS-I and DAIDALOS-II (Designing Advanced network Interfaces for the Delivery and Administration of Location independent, Optimized personal Services) European funded projects. Since 2006 he joined Portugal Telecom Inovação, Aveiro, Portugal, and he is involved in the WEIRD (WiMAX Extension to Isolated Research Data networks) and HURRICANE (Handovers for Ubiquitous and optimal bRoadband connectivity CooperAtive Networking Environments) European funded projects. Furthermore, since 2007 he is also pursuing a Ph.D. in Telecommunications and Informatics Engineering at the University of Aveiro, Portugal. He has been involved in two book chapters ("Advances in Mobile WiMAX" and "Evolving WiMAX") for Wiley, as well as more than 20 scientific papers in major journals and international conferences. His research interests are focused on broadband wireless access technologies, mobility and QoS management in all-IP heterogeneous environments, multicast and broadcast services, as well as mesh networks.

Paulo Simoes is an Auxiliary Professor at the Department of Informatics Engineering of the University of Coimbra, Portugal, from where he graduated in 1993 and got a PhD in Informatics Engineering on the subject of Distributed Network Management, in 2002. His research interests are Computer Communications, Mobility, Network and Systems Management and Security. He has participated in several national projects founded by the Foundation for Science and Technology, in Networks of Excellence from the $5^{\circ} \mathrm{FP}$ and $6^{\circ} \mathrm{FP}$, and on the $6^{\circ} \mathrm{FP}$ Integrated Projects EuQoS - End-to-end Quality of Service support over Heterogeneous Networks - and WEIRD - WiMax Extensions to Isolated Research Data Networks. 

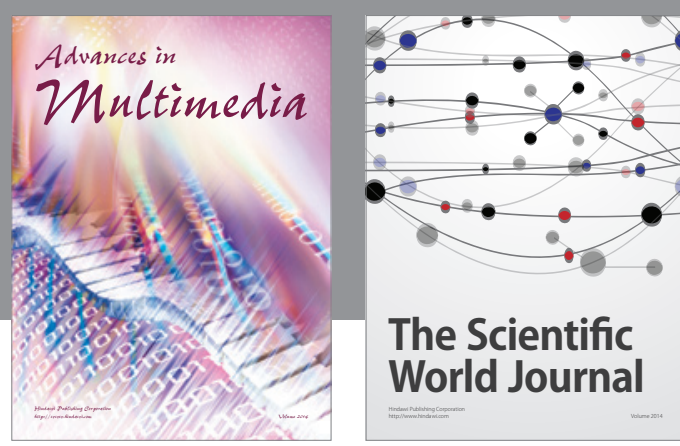

The Scientific World Journal
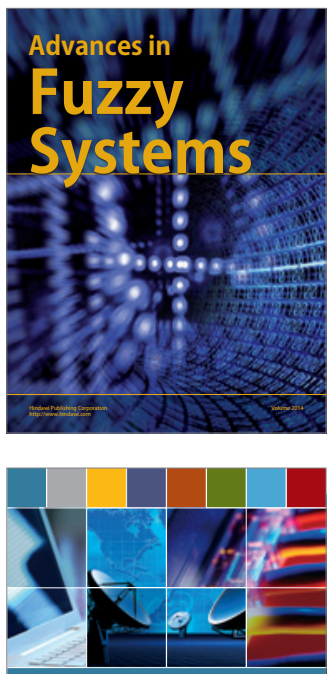

Computer Networks and Communications
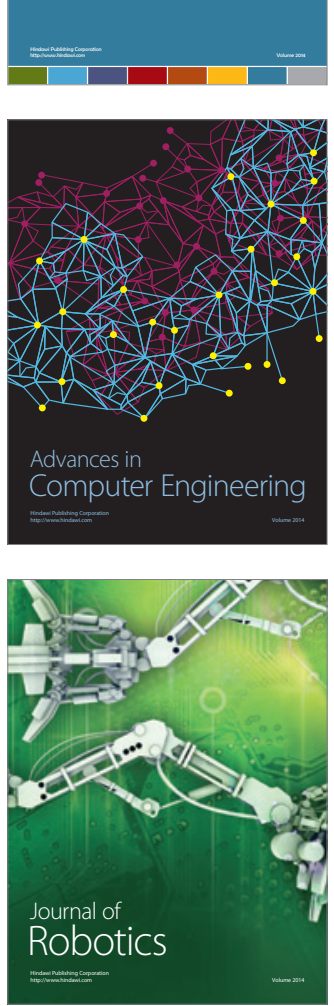
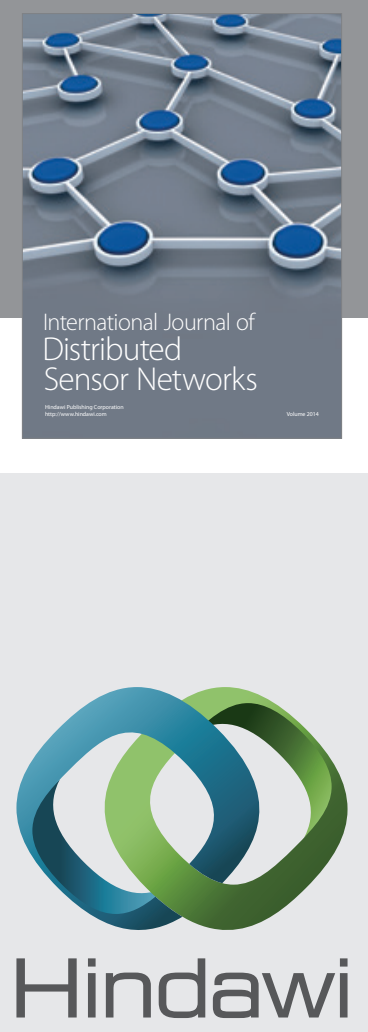

Submit your manuscripts at

http://www.hindawi.com
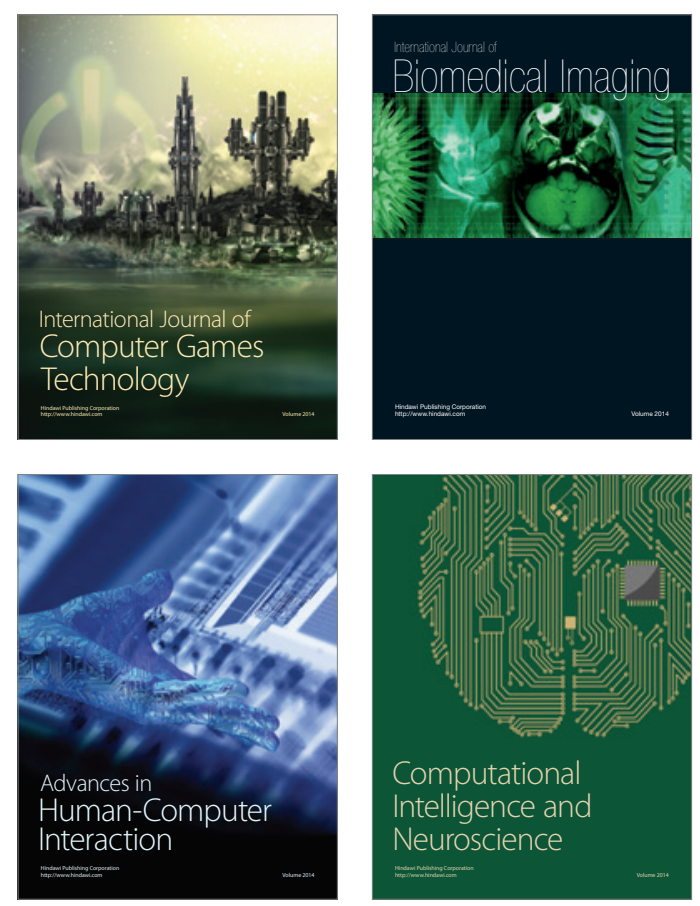
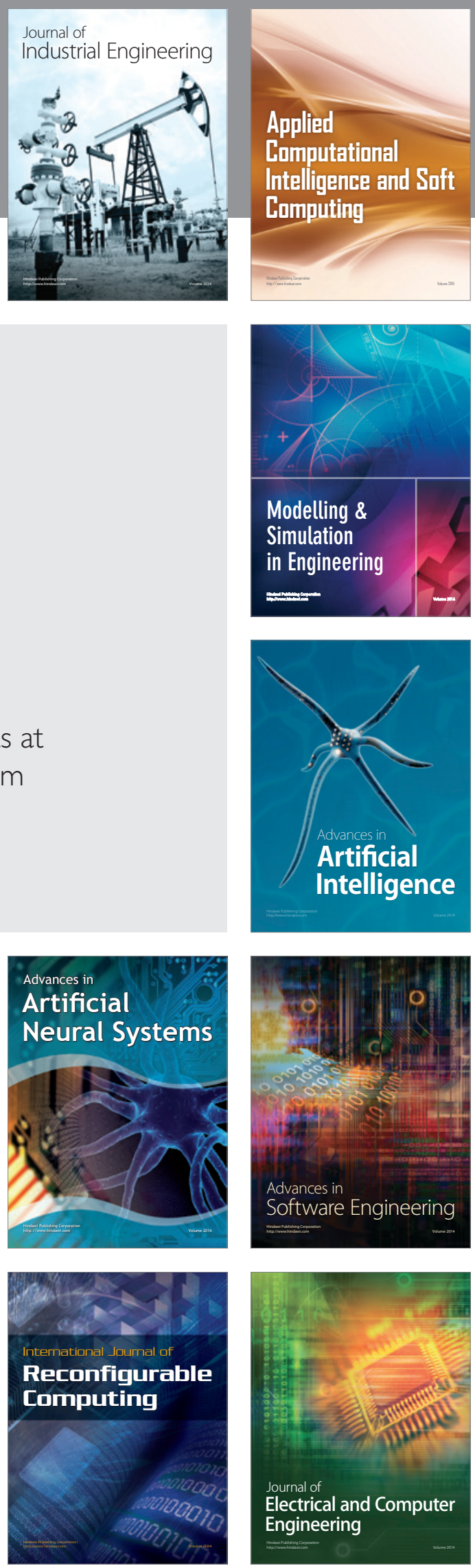